\title{
KRAB-independent Suppression of Neoplastic Cell Growth by the Novel Zinc Finger Transcription Factor KS1
}

\author{
Brian Gebelein, ${ }^{\star}$ Martin Fernandez-Zapico, ${ }^{\ddagger}$ Mami Imoto, ${ }^{\ddagger}$ and Raul Urrutia ${ }^{\star \ddagger}$ \\ ${ }_{*}^{*}$ Department of Molecular Neuroscience, Gastroenterology Research Unit, ${ }^{\ddagger}$ Saint Marys Hospital, and ${ }^{\S}$ Department of Biochemistry and \\ Molecular Biology, Mayo Clinic, Rochester, Minnesota 55905
}

\begin{abstract}
The study of zinc finger proteins has revealed their potential to act as oncogenes or tumor suppressors. Here we report the molecular, biochemical, and functional characterization of KS1 (KRAB/zinc finger suppressor protein 1), a novel, ubiquitously expressed zinc finger gene initially isolated from a rat pancreas library. $\mathrm{KS} 1$ contains $10 \mathrm{C}_{2} \mathrm{H}_{2}$ zinc fingers, a KRAB-A/B motif, and an ID sequence that has been shown previously to participate in growth factor-regulated gene expression. Northern blot analysis using pancreatic cell lines demonstrates that KS1 mRNA is inducible by serum and epidermal growth factor, suggesting a role for this gene in cell growth regulation. Biochemical analysis reveals that KS1 is a nuclear protein containing two transcriptional repressor domains, $\mathrm{R} 1$ and $\mathrm{R} 2$. $\mathrm{R} 1$ corresponds to the KRAB-A motif, whereas $\mathrm{R} 2$ represents a novel sequence. Transformation assays using NIH3T3 cells demonstrate that KS1 suppresses transformation by the potent oncogenes Ha-ras, $\mathrm{G} \alpha 12$, and $\mathrm{G} \alpha \mathbf{1 3}$. Deletion of the R1/ KRAB-A domain does not modify the transformation suppressive activity of KS1, whereas deletion of $\mathrm{R} 2$ abolishes this function. Thus, KS1 is a novel growth factor-inducible zinc finger transcriptional repressor protein with the potential to protect against neoplastic transformation induced by several oncogenes. (J. Clin. Invest. 1998. 102:1911-1919.) Key words: Krüppel-associated box • zinc finger $\bullet$ epidermal growth factor • exocrine pancreas $\bullet$ neoplastic transformation
\end{abstract}

\section{Introduction}

Recent studies on the molecular mechanisms underlying neoplastic transformation suggest that most human cancers result from alterations in pathways that regulate cell growth and differentiation in normal cells (1). Proteins that participate in cellular cascades that favor tumor development have received the name oncogenes, whereas those that normally inhibit neoplastic transformation are known as tumor suppressors (1). Thus, the discovery of these proteins has simplified the search for mechanisms underlying the origin of human tumors by focus-

Address correspondence to Dr. Raul Urrutia, Gastroenterology Research Unit, 2-445 Alfred Bldg., Saint Marys Hospital, Rochester, MN 55905. Phone: 507-284-7500; FAX: 507-255-6318; E-mail: urrutia.raul@mayo.edu

Received for publication 2 October 1997 and accepted in revised form 6 October 1998.

J. Clin. Invest.

(C) The American Society for Clinical Investigation, Inc. 0021-9738/98/12/1911/09 \$2.00

Volume 102, Number 11, December 1998, 1911-1919

http://www.jci.org ing on the identification of molecules that, when mutated or aberrantly expressed, lead cells to differentiate abnormally, adopt unregulated proliferative properties, or fail to undergo apoptosis.

Several laboratories, including ours, have focused on characterizing members of the $\mathrm{C}_{2} \mathrm{H}_{2}$ zinc finger transcription factor family as candidates for oncogenes or tumor suppressors. Many of these transcription factors play a key role in the regulation of cell proliferation, differentiation, and apoptosis in response to a variety of stimuli including growth factors, hormones, and extracellular matrix components (2-5). Because of their role in these pathways, some zinc finger transcription factors are powerful regulators of normal morphogenesis during embryonic development (4). Furthermore, mutations in some of these genes give rise to neoplastic transformation. For instance, mutations in the human WT1 gene have been demonstrated to be responsible for the development of Wilms' tumor (6). Other well-characterized examples of zinc finger transcription factors that are involved in neoplastic transformation include Evi1, Gli1, Lot1, and Bcd (7-10). Together, these data support a role for zinc finger proteins not only as regulators of normal morphogenesis, but also in the molecular mechanisms underlying the development of cancer.

Zinc finger proteins constitute a well-characterized group of transcription factors that can bind promoter sequences to activate and/or repress gene expression (11-17). Members of this family of transcription factors bind DNA through their $\mathrm{C}_{2} \mathrm{H}_{2}$ zinc finger domains, which are often found in tandem repeats with a variable number of units (18). Structural analyses have revealed that each finger behaves as an independent trinucleotide binding unit, while the variation in primary structure and number of fingers provides specificity for sequencespecific DNA recognition (18). In addition, zinc finger proteins can be divided into subfamilies based upon the presence of conserved structural motifs located outside of their DNAbinding domains. These motifs have often been found to directly regulate transcription. For instance, the KRAB motif is present in one-third of all $\mathrm{C}_{2} \mathrm{H}_{2}$ zinc finger proteins and can behave as a strong transcriptional repressor domain (14-17, 19). Therefore, these data indicate that zinc finger transcription factors contain independent protein modules for regulating different functional aspects of gene expression. Furthermore, the ability of several zinc finger proteins to regulate transcription has been shown to be required for their function in morphogenesis and neoplastic transformation (20-22).

In this study, we report the identification, nuclear localization, transcriptional regulatory properties, and transformation suppressive effects of KS1 (KRAB/zinc finger suppressor protein 1$),{ }^{1}$ a ubiquitously expressed gene that encodes a novel

1. Abbreviations used in this paper: ID, identifier; KS1, KRAB/zinc finger suppressor protein 1 . 
KRAB-containing zinc finger protein. The KS1 protein is localized to the nucleus and contains two potent transcriptional repression domains, $\mathrm{R} 1$ and $\mathrm{R} 2$. The $\mathrm{R} 1$ domain corresponds to the conserved KRAB-A motif, whereas R2 represents a novel repressor domain. Interestingly, when overexpressed in NIH3T3 cells, KS1 significantly suppresses transformation induced by several oncogenes, an activity that requires the R2 domain but not the R1/KRAB-A motif. Thus, these results demonstrate that KS1 harbors the potential to protect against neoplastic transformation, defines a role for the novel R2 repressor domain in the suppression of transformation, and suggests that KRAB domains are unlikely to modulate neoplastic cell growth.

\section{Methods}

Library screening and plasmid constructs. The KS1 cDNA was isolated from a cerulein-treated rat pancreas cDNA library (Stratagene, La Jolla, CA) using the DZF6 zinc finger probe previously identified from an exocrine pancreatic cell line (23). Library screening, DNA sequencing, and database analyses were performed as previously described (24). For immunofluorescence and transformation studies, the full-length KS1 and deletion mutants were cloned into the pcDNA3.1/HisA vector (InVitrogen, Carlsbad, CA) and sequenced. The KS1-GST fusion protein was generated using the pGEX4T-3 vector and purified using glutathione Sepharose 4B affinity chromatography (Pharmacia, Piscataway, NJ). KS1-GAL4 constructs were generated by amplifying various regions of KS1 by PCR and cloning these in-frame with the pSG424 GAL4 DBD effector plasmid (kindly provided by Dr. N.J. Zeleznik-Le, University of Chicago, Chicago, IL). The GAL4 ${ }_{5}$ tkCAT reporter vector was generously provided by Dr. N.J. Zeleznik-Le, the Ha-ras plasmid was a gift from Dr. E. San-

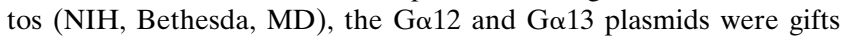
from Dr. L. Vitale-Cross (NIH, Bethesda, MD), the v-Src plasmid was kindly provided by Dr. S. Oldham (University of North Carolina, Chapel Hill, NC), and the WT1 expression vector was provided by Dr. D. Haber (MGH Cancer Center, Harvard Medical School, Cambridge, MA).

Cell culture, RNA isolation, and quantitative Northern blot analysis. The AR42J, AR4IP, PANC1, and NIH3T3 cell lines were obtained from the American Type Culture Collection (Rockville, MD) and cultured under an atmosphere containing $5 \% \mathrm{CO}_{2}$ in DMEM plus $5 \%$ FBS and $5 \%$ newborn calf serum. For the experiments on the regulation of KS1 gene expression by EGF, AR42J cells were cultured in serum-free DMEM for $24 \mathrm{~h}$ and subsequently treated with 5 $\mathrm{ng} / \mathrm{ml}$ murine EGF (GIBCO BRL, Gaithersburg, MD). $1 \mu \mathrm{g} / \mathrm{ml}$ actinomycin D (Sigma Chemical Co., St. Louis, MO) or $10 \mu \mathrm{g} / \mathrm{ml}$ cycloheximide (Sigma Chemical Co.) was applied 30 min before EGF stimulation for the early response experiments. Total RNA extraction and Northern blot analyses were performed as described previously (25). Blots were hybridized with the following probes: full-length $\mathrm{KS} 1$, ras, fos, or EGR $\alpha$. The probes for ras and EGR $\alpha$ were generated as described previously $(26,27)$. The fos probe spanning nucleotides 1560-1902 of the rat c-fos gene was generated by PCR (GenBank accession number X06769).

Generation of antibodies, Western blot analysis, immunofluorescence, and confocal microscopy. Antibodies against KS1 were generated in rabbits as described previously using a peptide corresponding to residues 546-561 (24). Whole cell lysates from AR42J cells and pancreatic tissues were extracted and Western blot analysis was performed as described previously (24). Immunolocalization of KS1 was performed using Xpress ${ }^{\mathrm{TM}}$ epitope-tagged constructs in methanolfixed PANC1 or NIH3T3 cells (GIBCO BRL) as described previously (28). The immunoreactivity corresponding to KS1 was visualized using a fluorescein-conjugated secondary antibody and the cytoplasm was contrasted using a computer-generated red pseudocolor.
GAL4-based transactivation assays and immunoprecipitation studies. GAL4-based transcriptional regulatory assays were performed using $3 \times 10^{5}$ PANC1 cells in $60-\mathrm{mm}$ tissue culture dishes transfected with $3 \mu \mathrm{g}$ effector plasmid DNA, $1 \mu \mathrm{g}$ GAL4 ${ }_{5}$ tkCAT reporter, and $1 \mu \mathrm{g}$ pHook-LacZ (InVitrogen) using LipofectAMINE ${ }^{\mathrm{TM}}$ (GIBCO BRL). CAT ELISA assays and quantitative analyses were performed as described previously (3). For immunoprecipitation studies, PANC1 cells were transfected in 100-mm tissue culture dishes with LipofectAMINE ${ }^{\mathrm{TM}}$ (GIBCO BRL). $48 \mathrm{~h}$ after transfection, cells were incubated for $1 \mathrm{~h}$ in methionine-free DMEM media containing $5 \%$ dialyzed FBS. The cells were then incubated in the same media containing $100 \mu \mathrm{Ci}\left[{ }^{35} \mathrm{~S}\right]$ methionine for $3 \mathrm{~h}$. Proteins were harvested in $3 \mathrm{ml}$ of lysis buffer $(150 \mathrm{mM} \mathrm{NaCl}, 0.5 \% \mathrm{NP}-40,50 \mathrm{mM}$ Tris, $\mathrm{pH} 7.5,20 \mathrm{mM} \mathrm{MgCl} 2,10 \mu \mathrm{g} / \mathrm{ml}$ aprotinin, and $0.5 \mathrm{mM}$ PMSF). Immunoprecipitation using an agarose-conjugated anti-GAL4 DBD antibody (sc-510 AC) was performed according to manufacturer's suggestions (Santa Cruz Biotechnology, Santa Cruz, CA). The immunoprecipitate was analyzed by SDS-PAGE and autoradiography using X-Omat film (Eastman Kodak Co., New Haven, CT).

Transformation and apoptosis assays. The transformation of NIH3T3 cells was assayed using a foci formation assay and a soft agar colony formation assay (29). $10^{6}$ cells in a $100-\mathrm{mm}$ tissue culture dish were transfected using LipofectAMINE ${ }^{\mathrm{TM}}$ (GIBCO BRL). Cells were transfected with $5 \mu \mathrm{g}$ of either pcDNA 3.1/HisA, KS1, or WT1 with or without Ha-Ras DNA $(0.25 \mu \mathrm{g}), \mathrm{G} \alpha 12(0.5 \mu \mathrm{g}), \mathrm{G} \alpha 13(0.5 \mu \mathrm{g})$, or $\mathrm{v}-\mathrm{Src}(0.5 \mu \mathrm{g})$. The total amount of DNA was equalized to $10 \mu \mathrm{g}$ using the pcDNA 3.1/HisA control plasmid. $24 \mathrm{~h}$ after transfection, cells from each dish were transferred into three $100-\mathrm{mm}$ tissue culture dishes. The media was changed every $2 \mathrm{~d}$, cells were stained with $0.4 \%$ crystal violet after $14 \mathrm{~d}$, and foci were counted as described (29). Each experiment was performed in triplicate in at least three different experiments, and the mean standard error was calculated. For soft agar assays, $2 \times 10^{4}$ transfected cells were resuspended in serumcontaining DMEM media with $0.33 \%$ low melting point agarose and plated over a cushion of $0.5 \%$ agarose in the same medium in a 60 $\mathrm{mm}$ dish (29). The media was changed every $4 \mathrm{~d}$ and colonies that formed after 3 wk of growth were scored using an inverted microscope. Three different experiments were performed in triplicate, and statistical analyses were performed using a Student's $t$ test. Apoptosis studies were performed using the terminal deoxynucleotidyl transferase-mediated dUTP nick end-labeling technique (TUNEL) (Boehringer Mannheim, Indianapolis, IN) and the annexin $\mathrm{V}$ technique (Clontech, Palo Alto, CA) as described $(2,30)$.

\section{Results}

Molecular characterization of KS1, a novel growth factor-inducible member of the KRAB subfamily of zinc finger-encoding genes. We report the identification and characterization of a novel growth factor-inducible KRAB-containing zinc fingerencoding gene, KS1. The KS1 cDNA was isolated from a cerulein-treated rat pancreas library using the 350-bp zinc finger probe, DZF6, that was amplified by PCR from a tumoral pancreatic cell line using degenerate primers for $\mathrm{C}_{2} \mathrm{H}_{2}$ transcription factor proteins as described previously (23). The KS1 cDNA was chosen for further study because it was inducible by serum and EGF in pancreatic cell lines, suggesting a role for this gene in the regulation of cell growth (Fig. 1). Using this approach, three identical KS1 cDNA clones were isolated from a rat pancreas cDNA library from a total of $2 \times 10^{6}$ plaques. Each of these cDNAs was $2.8 \mathrm{~kb}$ in length and contained a single open reading frame encoding a polypeptide of 566 amino acids with a calculated molecular mass of $\sim 65.5 \mathrm{kD}$ (Fig. 2). Sequence analyses and database comparisons shown in Figs. 2 and 3 indicate that KS1 contains several molecular motifs char- 


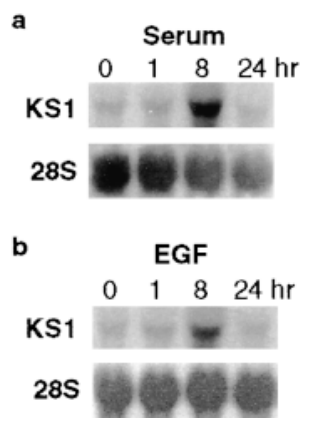

Figure 1. The expression of KS1 is upregulated by serum and EGF in rat exocrine pancreatic AR42J cells. $10 \mu \mathrm{g}$ of total RNA from AR42J cells treated with serum or EGF was analyzed by Northern blot analysis. Each experiment was performed at least two times with similar results. AR42J cells were serum-starved for $24 \mathrm{~h}$ and subsequently treated with $10 \%$ serum $(a)$ or $5 \mathrm{ng} / \mathrm{ml}$ murine $\mathrm{EGF}(b)$ for the indicated times. Both serum and EGF increased the levels of KS1 mRNA after $8 \mathrm{~h}$ of treatment. Control hybridizations for $28 \mathrm{~S}$ rRNA were performed as described previously (23).

acteristic of transcription factor proteins, including 10 different $\mathrm{C}_{2} \mathrm{H}_{2}$ zinc finger domains, and both a KRAB-A and a KRAB-B domain. The zinc finger motifs ( $\mathrm{ZF} 1$ to $\mathrm{ZF} 10$ ) match the consensus sequence for members of the $\mathrm{C}_{2} \mathrm{H}_{2}$ family of DNAbinding proteins (Fig. $3 a$ ) (4). Nine of the zinc finger domains are clustered at the $\mathrm{COOH}$ terminus of the KS1 protein, whereas a 56-amino acid spacer separates an additional $\mathrm{NH}_{2}-$ terminal zinc finger motif. In addition, a vestigial zinc finger, which is similar to the type previously described by Schulz et al. (31) and to a vestigial zinc finger of another pancreatic protein (GenBank accession number U56862), is located within the 56-amino acid spacer region. Interestingly, KS1 also possesses consensus KRAB-A and KRAB-B motifs, highly conserved sequences which are present in approximately one-

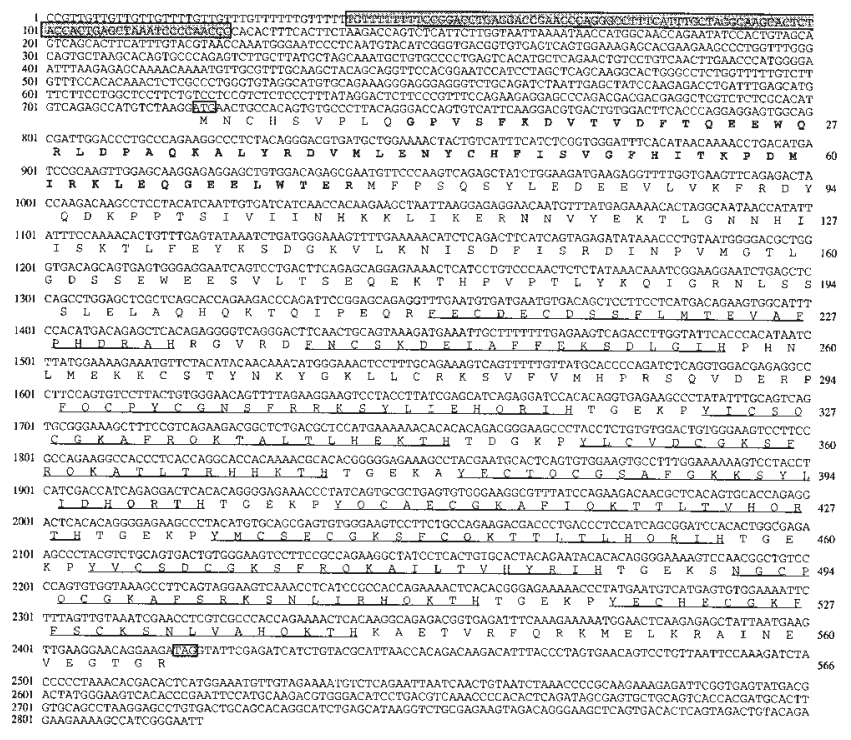

Figure 2. Nucleotide and deduced amino acid sequences of the rat KS1 encoding cDNA. KS1 encodes a polypeptide of 566 amino acids containing several structural domains previously demonstrated to be present in transcription factors from the KRAB subfamily of zinc finger proteins $(14-16,19) .10$ different $\mathrm{C}_{2} \mathrm{H}_{2}$ zinc finger domains and a vestigial zinc finger are underlined, and both a KRAB-A and a KRAB-B domain are shown in bold. The shaded box indicates the ID repeat within the $5^{\prime}$ untranslated region of this cDNA. The nucleotide sequence is numbered on the left, the deduced amino acid sequence is numbered on the right, and the initiation ATG and termination TAG codons are boxed. The KS1 GenBank/EMBL accession number is U56732. third of $\mathrm{C}_{2} \mathrm{H}_{2}$ zinc finger proteins (19) (Fig. $3 \mathrm{~b}$ ). In addition, the $5^{\prime}$ untranslated region of the KS1 cDNA contains an 85-bp sequence that is $85 \%$ identical to the highly repetitive brain identifier (ID) motif (Fig. 3c), a sequence previously reported in several mRNAs which are upregulated by serum and oncogenic Ha-ras in rat fibroblasts (32). Lastly, we determined the tissue distribution of the KS1 mRNA by Northern blot analysis and found that this gene is not only expressed in the pancreas, but is ubiquitously detected in adult rat tissues with the highest levels in lung, kidney, and testis (Fig. 4).

$K S 1$ is a nuclear protein that displays transcriptional regulatory activity. The sequence analysis of KS1 suggests a role for this protein as a transcription factor. Since transcription factors are localized to the nucleus to regulate gene expression, we used an epitope-tag approach to determine the intracellular localization of KS1. For these experiments, we expressed an epitope-tagged form of the KS1 protein in the highly transfectable exocrine pancreatic cell line, PANC1, and determined its localization by indirect immunofluorescence using confocal microscopy. Fig. 5 shows that KS1 localizes to the nucleus in these cells. PANC1 cells that were serum-starved for $24 \mathrm{~h}$ and then stimulated with serum or EGF from $10 \mathrm{~min}$ to $4 \mathrm{~h}$ had the same intracellular distribution of KS1, suggesting that this zinc finger protein is constitutively localized to the nucleus (data not shown).

To test whether the KS1 protein contains a transcriptional regulatory domain, we used the GAL4-based system. This assay is the most commonly used method for analyzing the functions of transcription factor proteins in mammalian cells (33, 34). For these studies, we constructed several deletions encompassing the $\mathrm{NH}_{2}$-terminal and the $\mathrm{COOH}$-terminal regions of $\mathrm{KS} 1$. These fragments were independently fused to the heterologous DNA-binding motif of the yeast GAL4 transcription factor and transfected into PANC1 cells together with a reporter plasmid containing five GAL4-binding sequences upstream of the CAT reporter gene. The expression of the CAT reporter was then determined by an ELISA-based assay. Fig. 6 shows that the $\mathrm{NH}_{2}$-terminal domain of $\mathrm{KS} 1$ behaves as a strong transcriptional repressor motif, whereas the $\mathrm{COOH}$ terminus displays no significant transcriptional regulatory activity (effector plasmids 2 and 7, respectively, Fig. 6). To better map the transcriptional repressor activity within the $\mathrm{NH}_{2}$-terminal region of KS1, we performed additional deletion analysis. This approach identified two potent repression domains that we call R1 and R2. The R1 domain of KS1, which contains the KRAB-A motif (residues 10-51), represses the expression of the CAT reporter by $>80 \%$ (effector plasmid 4, Fig. 6). In contrast, the KRAB-B domain (residues 52-74), also a conserved structural feature found in KRAB containing zinc finger proteins, does not modulate transcription in this assay (effector plasmid 5, Fig. 6). The second repression domain, R2, spans amino acids 75-210 and represses the expression of the CAT reporter activity by $>70 \%$ (effector plasmids 6, Fig. 6). Control immunoprecipitation studies indicate that all of these proteins are expressed at similar levels (Fig. $6 c$ ). These functional data demonstrate that KS1 contains two potent transcriptional repressor domains. Together with the sequence analysis and nuclear localization, these results strongly support a role for this protein as a transcription factor.

Temporal regulation of KS1 expression in response to $E G F$. A more defined temporal pattern of expression of KS1 in response to EGF was determined using Northern blot anal- 
ZF1 EECDEWDSSTMTEVAFPWDRAWKGVRD VZF FNESKDEIAFFEKSDAGIMPHNLMEKKC ZF2 FQCPY:GGNSFRRKSY I. IEVORIWTGEKP

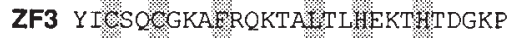
ZF4 YLCVKEGKS RERATITRIHKTHTGEKA ZF5 YECTOEGSAFGKKSYM IDIORT WTGEKP ZF6 YQCAECGKAFIQKTTI TVHORTITTEKP ZF7 YMESEFGKSWCOKTTIT TIHORIMTGEKP ZF8 YVESDKGKS KRQKAII TVIXYRITTGEKS ZF9 NGGPQGGKAFSRKSNIIRIOKTHTGERP ZF10 YECHEOGKFFSCKSN VAKOKTMKAETV Cons C..C...F....I..H...H

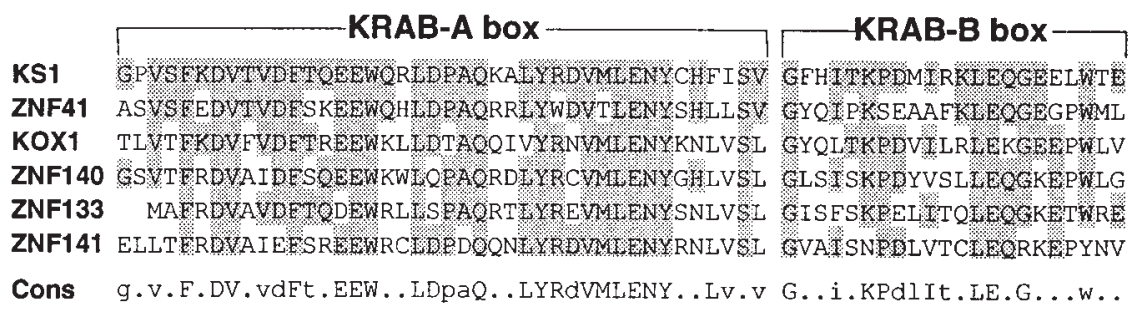

\section{ID Repeat}

KS1 125 GGGTTGGGGATTTAGCTCAGTGGTAGAGTGCTTGCCTAGCAAATGAAAGGCCCTGGGTTCGGTCCTCAGGTCCGGAAAAAAAACA 20

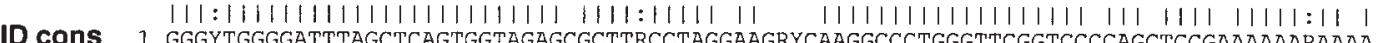

Figure 3. Sequence comparisons of structural domains found in KS1 with previously reported nucleotide and amino acid sequences. (a) Comparison of the KS1 zinc finger domains (ZF1-ZF10) and the $\mathrm{C}_{2} \mathrm{H}_{2}$ consensus sequence (Cons) (4). Identical amino acid residues of the consensus sequence are shaded. Note the vestigial zinc finger $(V Z F)$ of KS1 has several of the conserved amino acid residues of the consensus sequence but lacks a cysteine and histidine critical for coordinating a zinc ion (31). (b) Comparison of the KS1 KRAB-A and -B motifs with KRAB domains from other zinc finger proteins $(14-16,19)$. Identical residues are shaded. The KRAB-A and -B consensus sequence (Cons) is derived from Bellefroid et al. (19) where uppercase letters represent highly conserved residues, lowercase letters represent moderately conserved residues, and dots represent unconserved residues. (c) Comparison between the $5^{\prime}$ untranslated region ID repeat of KS1 and the consensus sequence for the ID motif (ID cons) described previously in growth factor-regulated mRNAs (32).

ysis. Fig. 7 a demonstrates that KS1 mRNA levels peak as early as $4 \mathrm{~h}$ after the treatment of pancreatic AR42J cells with EGF. Note that this early upregulation in KS1 mRNA expression occurs slightly later than that of the known immediate early response genes c-fos and $\operatorname{EGR} \alpha(27,35)$. However, the upregulation of KS1 still occurs in the presence of the protein synthesis inhibitor cycloheximide, suggesting that this gene belongs to the group of delayed early response genes (36). In contrast, treatment with the transcriptional inhibitor actinomycin D abolishes the upregulation of KS1 mRNA (Fig. $7 c$ ). Together, these results indicate that KS1 is a delayed early response target for EGF and that the effect of this growth factor on KS1 gene expression occurs at the level of transcription. To determine whether the upregulation of KS1 mRNA levels was accompanied by an increase in the KS1 protein, we also performed Western blot analysis using a polyclonal antibody raised against a region within the $\mathrm{COOH}$ terminus of the KS1 protein that is not shared with any previously described peptide deposited in existing data banks. This antibody specifically recognizes a recombinant GST-KS1 fusion protein and a protein of the expected molecular weight in adult rat pancreas homogenates (Fig. $8 a$ ). Furthermore, Fig. $8 b$ demonstrates that, similar to the KS1 mRNA, the level of the KS1 protein also increases after the stimulation of AR42J cells with EGF.

KS1 inhibits neoplastic cell growth in NIH3T3 cells. To determine if KS1 can induce neoplastic transformation like some previously described oncogenic zinc finger transcription factors, we assessed its ability to induce foci formation (anchorage-dependent cell growth) and colonies in soft agar (anchorage-independent cell growth) in NIH3T3 cells (29). The results in Fig. 9 demonstrate that KS1 is not transforming by itself in the foci formation assay. Subsequently, we used a Ha-rasbased transformation model to test whether KS1 displays an inhibitory effect on oncogene-mediated transformation, a fea-

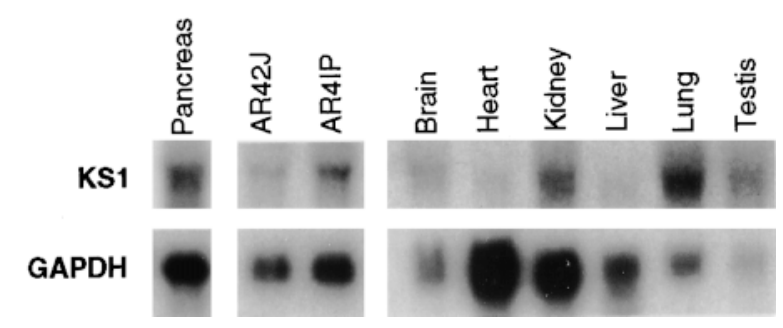

Figure 4. Expression of KS1 in adult rat tissues and exocrine pancreatic cell lines. $10 \mu \mathrm{g}$ of total RNA from adult rat tissues and the exocrine pancreatic cell lines AR4IP and AR42J were analyzed for KS1 expression by Northern blot analysis. Control hybridizations were performed using the glyceraldehyde-3-phosphate dehydrogenase $(G A P D H)$ gene (24). KS1 is ubiquitously expressed, with high levels found in pancreas, lung, kidney, testis, and brain.

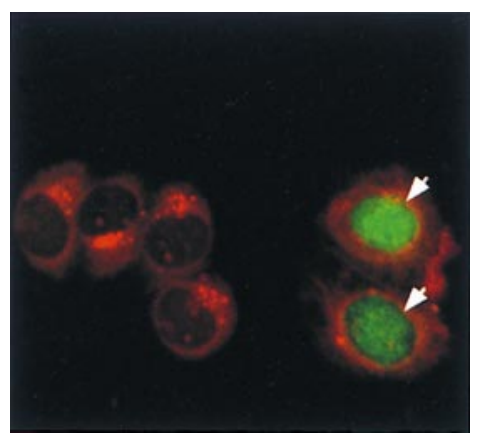

Figure 5. Nuclear localization of KS1 in the human exocrine pancreatic cell line, PANC1. Cells were transfected with an Xpress ${ }^{\mathrm{TM}}$ epitope-tagged KS1 vector, and localization was performed using a monoclonal anti-Xpress ${ }^{\mathrm{TM}}$ antibody and a fluoresceinconjugated anti-mouse secondary antibody. The arrowheads show nuclear staining of the KS1 construct in transfected PANC1 cells, whereas four untransfected cells in the field show no fluorescein staining. The cellular cytoplasm was pseudocolored in red to contrast nuclear versus cytoplasmic localization. 


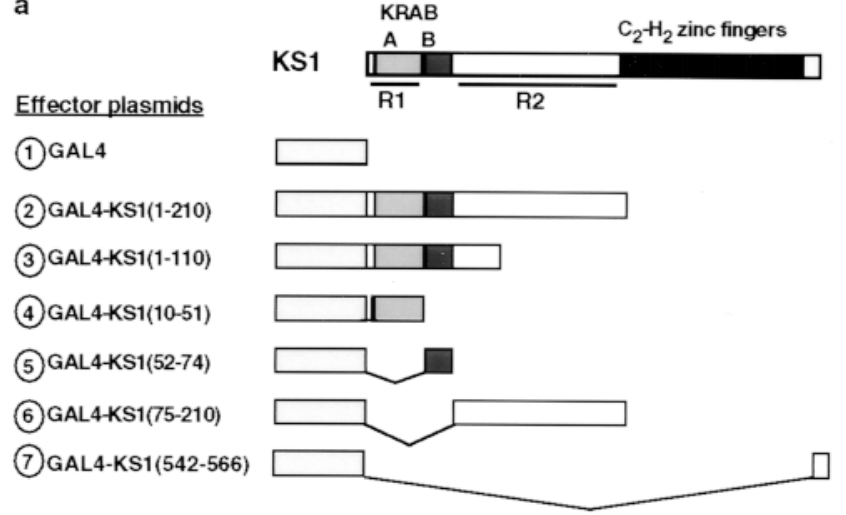

b
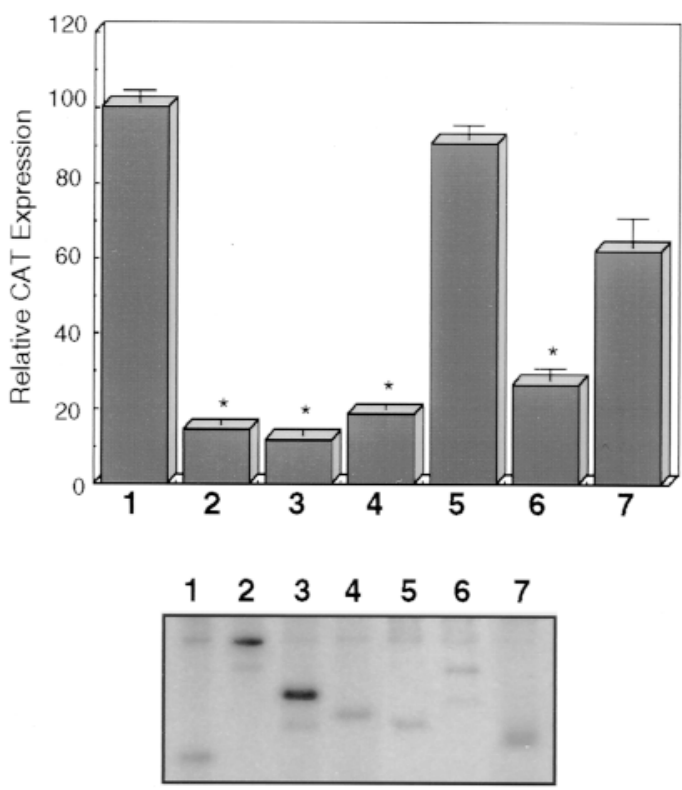

Figure 6. Transcriptional repressor activity of KS1. (a) Physical map of the GAL4/KS1 effector constructs used to cotransfect into PANC1 cells along with a CAT reporter plasmid containing five GAL4-binding sites. Basal transcriptional activity was measured using the reporter plus an effector plasmid carrying the GAL4 DNA-binding motif alone (construct 1). CAT expression was determined using an ELISA. For normalization purposes, the pHook-LacZ plasmid was cotransfected with each experimental condition. (b) Histogram of CAT expression demonstrates that the $\mathrm{NH}_{2}$ terminus (construct 2) of KS1 contains a strong transcriptional repressor domain, whereas the $\mathrm{COOH}$ terminus (construct 7) does not display any significant transcriptional regulatory activity versus the control. Deletion analysis of the $\mathrm{NH}_{2}$ terminus shows that KS1 encodes two transcriptional repressor domains, R1 (amino acids 10-51, construct 4) and R2 (amino acids $75-210$, construct 6 ). The asterisks denote that the CAT expression values are statistically significant from the GAL4 DBM construct alone $(P<0.001)$. (c) Immunoprecipitation of the GAL4/KS1 effector constructs from $\left[{ }^{35} \mathrm{~S}\right]$ methionine-labeled PANC1 cells was performed using an agarose-conjugated GAL4 antibody. SDS-PAGE and autoradiography demonstrate that these constructs are expressed at approximately equal levels.

ture previously described for several tumor suppressors, including the zinc finger protein WT1 (37). Fig. 9 demonstrates that the overexpression of KS1 in NIH3T3 cells significantly reduces the number of foci induced by an oncogenic Ha-ras a

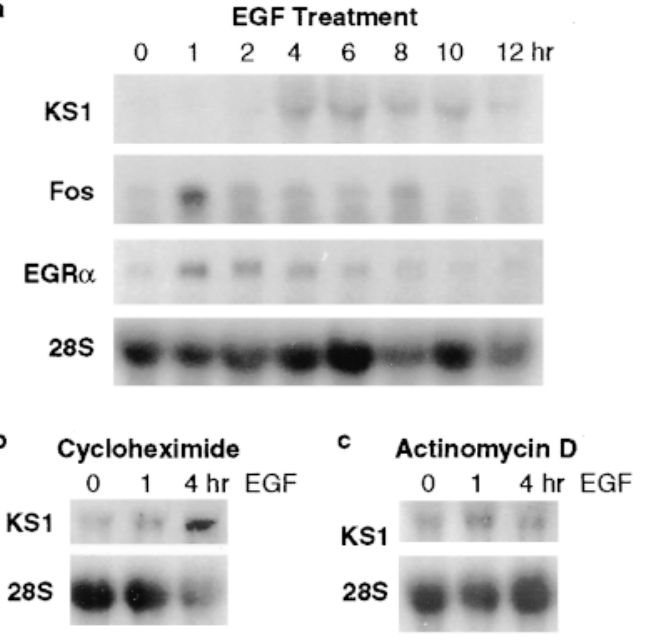

Figure 7. KS1 is a delayed early response gene for EGF. AR42J cells were serum-starved for $24 \mathrm{~h}$ and subsequently treated with $5 \mathrm{ng} / \mathrm{ml}$ EGF $(a), 5 \mathrm{ng} / \mathrm{ml} \mathrm{EGF}$ and $10 \mu \mathrm{g} / \mathrm{ml}$ cycloheximide $(b)$, or $5 \mathrm{ng} / \mathrm{ml}$ EGF and $1 \mu \mathrm{g} / \mathrm{ml}$ actinomycin D (c). EGF induces an upregulation in the expression of KS1 which peaks as early as $4 \mathrm{~h}$ after treatment $(a)$. Note that the upregulation of KS1 mRNA expression occurs slightly later than c-fos and $\mathrm{EGR} \alpha$, other early response genes $(27,35)$. Cycloheximide treatment does not effect the upregulation of KS1 mRNA levels $(b)$, whereas actinomycin D treatment abolishes this upregulation $(c)$. Northern blot analysis was performed as described in Methods. Each experiment was performed at least two times with similar results.

mutant when compared with cells transfected with Ha-ras alone. Interestingly, the inhibitory effect of KS1 on the number of foci formed by Ha-ras-transfected cells is similar to that of WT1 (Fig. 9). This inhibition of the ras-mediated anchorage-dependent growth was not due to decreased Ha-ras expression caused by cotransfection with $\mathrm{KS} 1$, as shown by Northern blot analysis in Fig. $9 c$. Furthermore, the inhibitory effect of KS1 on ras-induced foci formation is not due to an apoptotic effect of this transcription factor as assessed by the TUNEL labeling technique and annexin-V binding (data not shown). The inhibitory effect of KS1 on ras-mediated transformation was confirmed using a soft agar assay. Table I summarizes the results of these experiments which show that KS1 decreases the number of colonies formed in soft agar by oncogenic ras. Thus, KS1 inhibits not only ras-mediated anchoragedependent cell growth, but also anchorage-independent cell

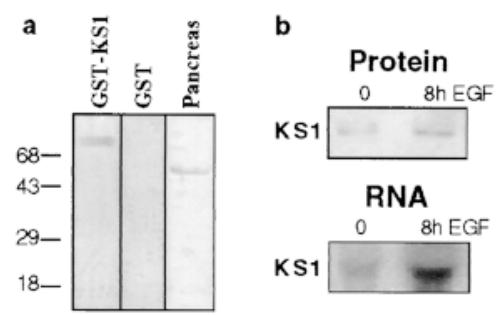

Figure 8. Upregulation of KS1 protein levels during EGF treatment of AR42J cells. (a) Western blot analysis of purified KS1GST fusion protein or GST alone. The KS1 antibody detects a $60-\mathrm{kD}$ peptide by Western blot analysis of whole pancreas homogenates from adult rats. $(b)$ Northern blot and Western blot analyses of EGF-treated AR42J cells. The upregulated expression of KS1 which occurs after $8 \mathrm{~h}$ of EGF treatment in AR42J cells by Northern blot analysis is also detected at the protein level with this antibody. RNA loaded $=10 \mu \mathrm{g} / \mathrm{lane}$; protein loaded $=40 \mu \mathrm{g} / \mathrm{lane} ;$ Ab dilution $=1: 1,000$. 
a
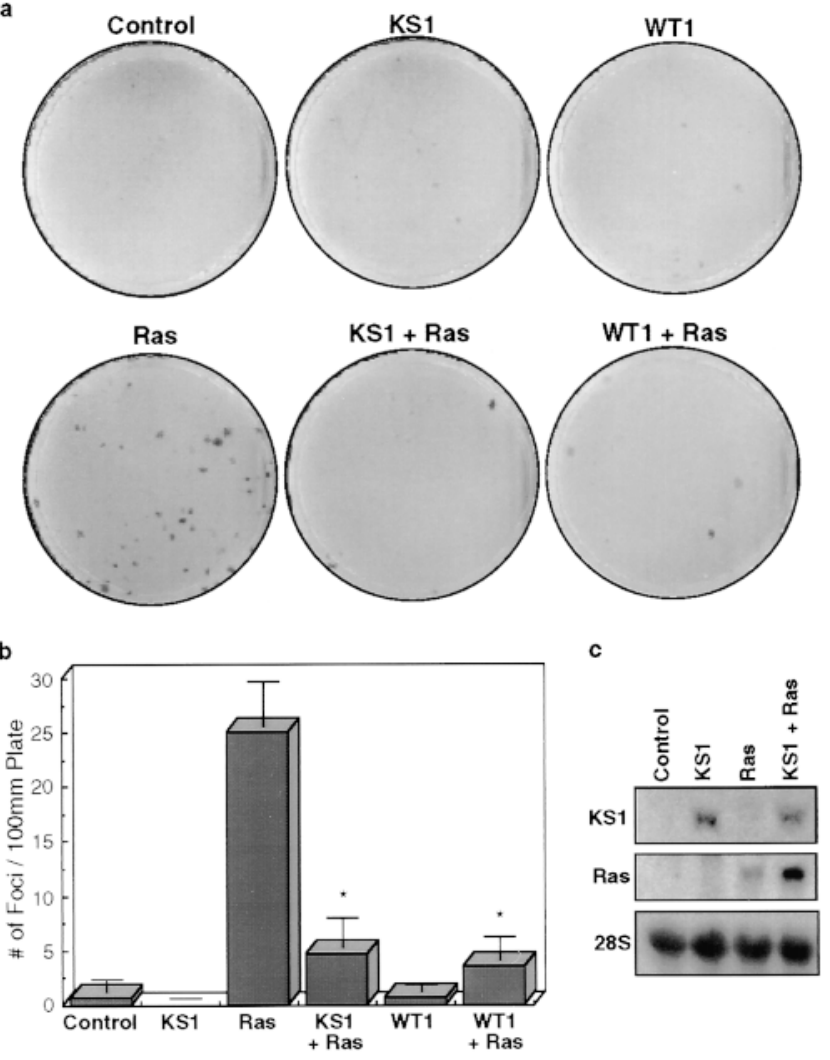

Figure 9. KS1 inhibits anchorage-dependent ras-mediated transformation of NIH3T3 cells. (a) Neoplastic transformation in NIH3T3 cells was assayed through an oncogenic ras foci formation assay (29). KS1 alone does not transform NIH3T3 cells. However, both KS1 and WT1 significantly inhibit the number of foci induced by Ha-ras. (b) Histogram showing that both KS1 and WT1 significantly inhibit Haras-induced foci formation $(P<0.01)$. Each experiment was performed in triplicate, and the mean standard deviation was calculated. (c) Control Northern blot analysis demonstrates that KS1-mediated suppression of transformation is not due to the inhibition of Ha-ras expression. NIH3T3 cells were transfected using the same protocol as for the foci formation assay and Northern blot analysis was performed as described in Methods.

growth. We also asked whether KS1 could inhibit transformation induced by other well-characterized oncogenes such as $\mathrm{G} \alpha 12$, G $\alpha 13$, and v-Src (38-40). The results of the foci formation assays shown in Fig. 10 demonstrate that KS1 significantly inhibits transformation by $\mathrm{G} \alpha 12$ and Go13, but not by v-Src. The inability of KS1 to inhibit v-Src-mediated transformation reveals that this protein displays some degree of selectivity for certain oncogenic cascades. Taken together, these results indicate that KS1 can function as a potent suppressor of neoplastic cell growth induced by several well-characterized oncogenes in vitro.

We next assessed the contribution of each KS1 transcriptional repression domain to the transformation suppressing activity of this protein using deletion mutants. cDNAs encoding KS1 proteins containing or lacking the $\mathrm{R} 1$ and $\mathrm{R} 2$ domains (Fig. 11a) were cotransfected into NIH3T3 cells together with the Ha-ras oncogene, and the number of foci formed was scored. Northern blot analysis was used to confirm that these constructs were expressed at similar levels (Fig. 11 b). Immu-
Table I. Growth of NIH3T3 Transfected Cells in Soft Agar Suspension Culture

\begin{tabular}{lc}
\hline Transfected cells & Number of colonies \\
\hline Control & $11.6 \pm 1.8$ \\
KS1 & $9.3 \pm 1.6$ \\
Ras & $58.4 \pm 6.3$ \\
Ras + KS1 & $34.2 \pm 3.1^{*}$
\end{tabular}

Cells transfected with KS1 and/or Ha-Ras were plated on 60-mm dishes in $0.33 \%$ low melting point agarose in DMEM as described in Methods. Colonies after 3 wk of growth were scored. Three different experiments were performed in triplicate. Values are the mean \pm standard error from one representative experiment. Statistical analyses were performed by Student's $t$ test. *Significant difference $(P<0.01)$ between the oncogenic ras-transfected cells and cells cotransfected with ras and KS1.

nofluorescence was used to determine that, similar to the fulllength $\mathrm{KS} 1$, all of the mutant proteins were localized to the nucleus of NIH3T3 cells (Fig. 11d). Fig. 11 $c$ shows that the deletion of both the R1 and R2 domains of KS1 results in the loss of the transformation suppressive activity. Interestingly, a KS1 protein containing the $\mathrm{R} 2$ domain but lacking the $\mathrm{R} 1$ domain retained full suppressive activity as compared with the fulllength KS1 protein. However, a construct containing the R1 domain but lacking the R2 domain of KS1 did not suppress ras transformation. Thus, these experiments demonstrate that the $\mathrm{R} 2$ domain but not the R1 domain of KS1 is necessary for the suppressive activity of this protein on cell transformation.

\section{Discussion}

In this study, we report the identification of KS1, a novel growth factor-inducible zinc finger transcription factor that suppresses neoplastic transformation of cultured NIH3T3 cells. KS1 was cloned as a result of our continuing efforts to

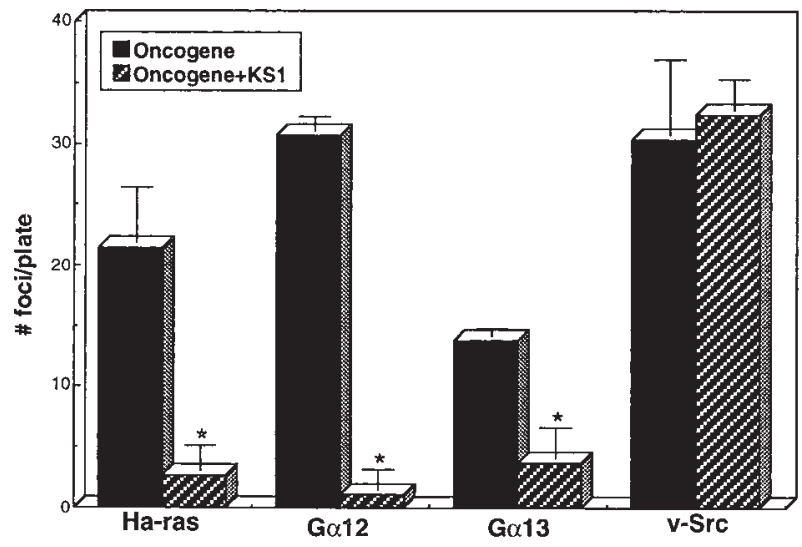

Figure 10. KS1 inhibits transformation induced by Ha-ras, G $\alpha 12$, and $\mathrm{G} \alpha 13$ oncogenes, but not by v-Src. Histogram showing the results of foci formation assays in NIH3T3 cells cotransfected with KS1 and either Ha-ras, G $\alpha 12, \mathrm{G} \alpha 13$, or v-Src. Experiments were performed as described in Fig. 9. Note that KS1 significantly inhibits foci formation (asterisks) by the $\mathrm{G} \alpha 12$ and $\mathrm{G} \alpha 13$ oncogenes $(P<$ 0.01 ), but not by the v-Src oncogene. 


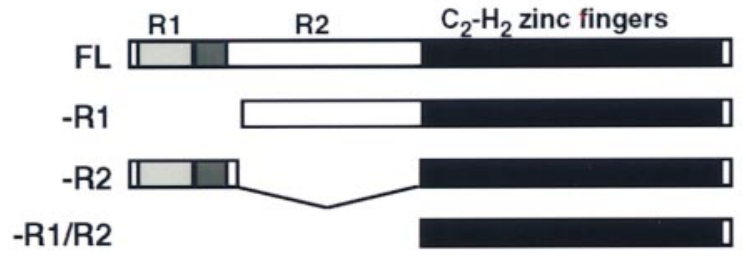

b

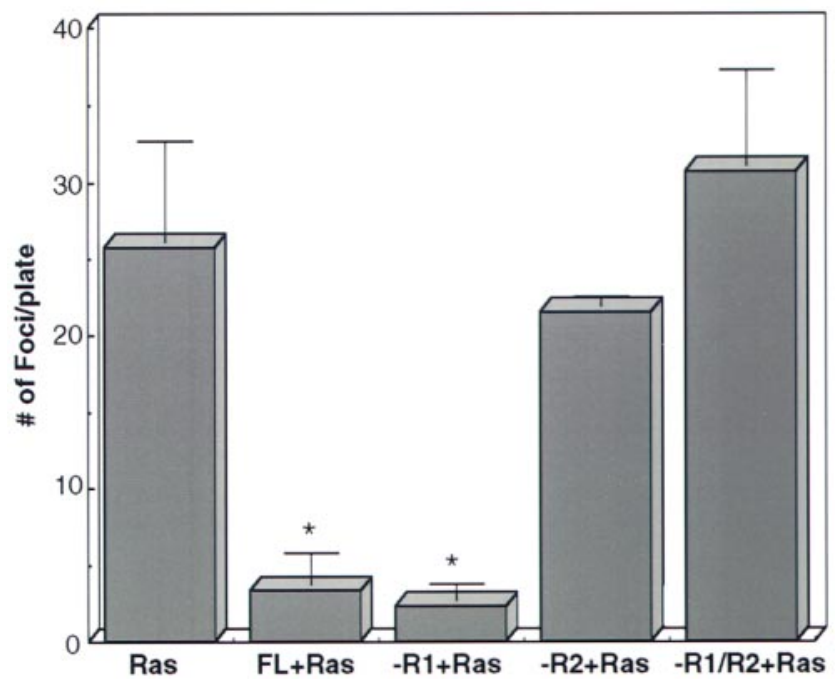

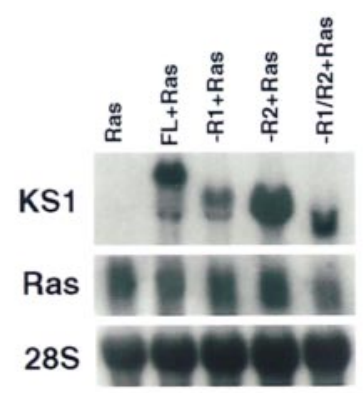

d
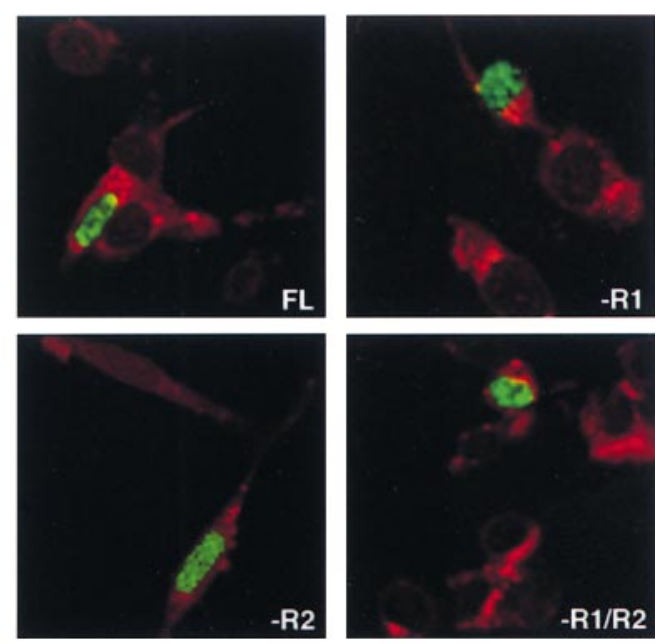

Figure 11. The R2 domain of KS1 is necessary to inhibit ras-mediated transformation. Foci formation assays were performed in NIH3T3 cells using KS1 deletion mutants to test whether the R1 and R2 transcriptional repressor domains are necessary for the transformation suppressing activity of this protein. (a) Diagram of KS1 constructs carrying deletions of the R1 and/or the R2 domains. Transformation activity was determined by scoring the number of foci formed by Ha-ras. (b) Histogram showing that full-length KS1 $(F L)$ decreases the number of Ha-rasinduced foci $($ Ras $)$. A construct containing the R2 domain but lacking the R1 domain $(-R 1)$ retained full suppressive activity. A construct containing the R1 domain but lacking the R2 domain of KS1 no longer displays transformation suppressive activity. Deletion of both the R1 and R2 domain of $\operatorname{KS1}(-R 1 / R 2)$ also abolishes the suppressive activity of this protein $\left({ }^{*} P<0.01\right)$. (c) Control Northern blot analysis, performed as described in Fig. 9, demonstrates that KS1-mediated suppression of transformation is not due to the inhibition of Ha-ras expression. (d) Indirect immunofluorescence localization demonstrates that, similar to full-length KS1, the transfected Xpress ${ }^{\mathrm{TM}}$ epitope-tagged KS1 deletion constructs are localized to the nucleus in these cells.

identify zinc finger transcription factors that can behave as either oncogenes or tumor suppressors. The detailed biochemical and functional characterization of KS1 was pursued because its inducibility by growth-regulatory peptides suggested that this protein may participate in growth regulatory pathways. Indeed, the data provided in this study demonstrate that KS1 has the potential to suppress neoplastic cell growth induced by several potent oncogenes.

Sequence analysis reveals that KS1 displays structural homology with other previously described $\mathrm{C}_{2} \mathrm{H}_{2}$ zinc finger proteins, which suggested that this protein may function as a transcription factor (4). To test this hypothesis, we determined whether KS1 is a nuclear protein using an epitope-tag approach and indirect immunofluorescence. Our results demonstrate that KS1, similar to other zinc finger transcription factors such as WT1, is constitutively localized to the cell nucleus $(41,42)$. Therefore, it is unlikely that translocation of KS1 from the cytoplasm into the nucleus is a rate-limiting step for the regulation of its function. We also determined the tran- scriptional regulatory ability of KS1 using a GAL4-based assay. This system has been used widely for characterizing the function of transcription factors in cultured cells, including several members of the zinc finger family of proteins (34). Our data demonstrate that the $\mathrm{NH}_{2}$ terminus of $\mathrm{KS} 1$ behaves as a potent transcriptional repressor domain in exocrine pancreatic cells. However, we did not detect any change in the repressor activity of this domain by the addition of $5 \mathrm{ng} / \mathrm{ml} \mathrm{EGF}$ for 1, 2, or $24 \mathrm{~h}$ (data not shown), indicating that it is unlikely that EGF directly modulates the transcriptional repressor activity of this protein. In contrast, it is more likely that EGF-mediated signaling cascades regulate the function of KS1 by upregulating its expression rather than through the modification of its intracellular localization or transcriptional regulatory properties. Further deletion analysis reveals the presence of two potent repression domains, $\mathrm{R} 1$ and $\mathrm{R} 2$, located within the $\mathrm{NH}_{2}$-terminal region of KS1. R1 spans a conserved KRAB-A motif, whereas $\mathrm{R} 2$ represents a novel repression domain located immediately upstream of the zinc finger region of this protein. In- 
terestingly, other KRAB-A domains have also been shown to display repressor activity in heterologous in vitro transcription factor assays (14-16). The activity of the KRAB-A motif is thought to function through the interaction with the bromodomain-containing protein KAP-1 (43-45). However, the R2 domain does not display significant homology with any previously described transcriptional regulatory domain, and thus, represents a newly identified repressor motif. Future characterization of proteins that potentially interact with this motif may provide insight into the mechanisms underlying its repressor activity.

Evidence for the ability of KS1 to modulate neoplastic cell growth was initially gathered through the use of both ras-mediated foci formation and soft agar colony formation assays. These assays have been fundamental for revealing the growthregulatory function of a large number of molecules (29). For instance, overexpression of members of the ras family of proteins plays a pivotal role in cell proliferation and has been shown to induce both foci and colony formation (29). In contrast, other molecules harboring antiproliferative potential, such as the WT1 zinc finger protein, inhibit ras-induced neoplastic cell growth in these assays (42). The expression of KS1 displays an inhibitory effect on both anchorage-dependent and -independent cell growth induced by ras, revealing its potential as a negative regulator of neoplastic transformation. Furthermore, this activity is not specific for ras since KS1 was also able to suppress the ability of two other potent oncogenes, G $\alpha 12$ and $\mathrm{G} \alpha 13$, to transform cells. Interestingly, however, KS1 does not inhibit Src-mediated transformation in the same assay, suggesting that KS1 inhibits downstream components of distinct signaling cascades with some degree of selectivity. This phenomenon of selectively inhibiting distinct oncogenic pathways has also been observed with other proteins such as p16INK4 which inhibits Ha-ras/Myc transformation but not Ha-ras/E1A transformation (46). These results also suggest that KS1 may inhibit a pathway that is shared by ras, G $\alpha 12$, and $\mathrm{G} \alpha 13$ to induce transformation. Future studies will focus on determining the identity of this pathway.

It is important to note that the transformation inhibitory activity that KS1 displays in these assays resembles that of well-characterized tumor suppressor genes. Tumor suppressor genes not only behave as negative regulators of cell growth, but in addition are often mutated in primary human tumors (1). A good example of such a protein is the WT1 gene product described above which inhibits neoplastic cell growth, and through mutations gives rise to Wilms' tumors $(6,42,47)$. Interestingly, in our hands, the ability of KS1 to suppress oncogene-induced foci formation was comparable to that of WT1, suggesting that these two zinc finger proteins display similar potentials to inhibit neoplastic transformation. Currently, however, we do not know whether KS1 is mutated or deleted in any type of cancer, data that would further support the idea that this gene functions as a tumor suppressor. Therefore, to extend this study, future experiments will focus on determining the integrity of this gene in a battery of different human primary tumors.

The experimental evidence reported here characterizes the structure, biochemical properties, and growth regulatory activity of KS1. An additional question to be addressed is: What are the molecular mechanisms used by KS1 to suppress neoplastic cell growth? In this regard, data derived from the analysis of other growth factor-regulated transcription factors such as
WT1 and EGR-1 allow us to hypothesize that the ability of KS1 to suppress transformation is related to its transcriptional regulatory properties $(5,20-22)$. For instance, like WT1 and EGR-1, KS1 may repress the expression of genes encoding mitogenic signaling proteins or cell cycle regulators. This mechanism may be used by growth factor-mediated signaling cascades as a security device that limits the extent of cell proliferation after they have been initiated. This mechanism has been defined previously for WT1, which can downregulate growth factor receptors and interact with p53 in order to regulate cell cycle arrest and programmed cell death (42). To determine whether the function of KS1 as a transcription factor contributes to its transformation suppressive activity, we performed foci formation assays using deletion mutant forms of this protein. The deletion of the entire $\mathrm{NH}_{2}$-terminal domain of KS1 abolished its suppressive activity, suggesting that the transcriptional repressor activity of KS1 contributes to the antitransformation function of this protein. Because the KRAB domains are present in almost a third of mammalian zinc finger proteins, we tested whether this domain was required for transformation. This is important since one member of this subfamily of proteins, ZNF74, has been proposed as a candidate for the DiGeorge syndrome, a disease characterized by a striking incidence of childhood tumors (48). Interestingly, however, a KRAB minus KS1 cDNA inhibits ras-mediated transformation with an efficiency comparable to full-length $\mathrm{KS} 1$. Thus, this result suggests that it is unlikely that KRAB domains modulate neoplastic transformation. In contrast, the deletion of the R2 repressor motif completely abolished the suppressive function of KS1. This result suggests a correlation between the biochemical properties of this region of KS1 and its antitransformation activities. Thus, future studies focused on identifying proteins that may interact with this domain may prove useful for further dissecting the molecular mechanisms underlying the suppressive activity of KS1.

In conclusion, we have identified KS1 as a novel growth factor-inducible gene encoding a member of the KRAB subfamily of zinc finger proteins. The fact that KS1 protein is localized to the nucleus and contains two transcriptional repressor domains strongly supports a role for this protein as a transcription factor. Furthermore, our experiments constitute the first functional study revealing the potential of this protein to protect against neoplastic transformation. Future studies will be aimed at better defining the mechanism by which KS1 inhibits transformation as well as determining the role of this gene as a tumor suppressor in human cancers.

\section{Acknowledgments}

This work was supported by the Mayo Foundation, a grant from The Fraternal Order of Eagles, and the Charlotte Geyer Foundation to R. Urrutia. M. Fernandez-Zapico was supported by a fellowship from the International Rotary Club of Rio Cuarto, Córdoba, Argentina.

\section{References}

1. Ruddon, R.W. 1995. Cancer Biology. Oxford University Press, New York. $520 \mathrm{pp}$

2. Tachibana, I., M. Imoto, P.N. Adjei, G.J. Gores, M. Subramaniam, T.C. Spelsberg, and R. Urrutia. 1997. Overexpression of the TGFß-regulated zinc finger encoding gene, TIEG, induces apoptosis in pancreatic epithelial cells. $J$. Clin. Invest. 99:2365-2374.

3. Cook, T., B. Gebelein, K. Mesa, A. Mladek, and R. Urrutia. 1998. Molecular cloning and characterization of TIEG2 reveals a new subfamily of trans- 
forming growth factor- $\beta$-inducible Sp1-like zinc finger-encoding genes involved in the regulation of cell growth. J. Biol. Chem. 273:25929-25936.

4. El-Baradi, T., and T. Pieler. 1991. Zinc finger proteins: what we know and what we would like to know. Mech. Dev. 35:155-169.

5. Gashler, A., and V.P. Sukhatme. 1995. Early growth response protein 1 (EGR-1): prototype of a zinc finger family of transcription factors. Prog. $\mathrm{Nu}$ cleic Acid Res. Mol. Biol. 50:191-224.

6. Call, K.M., T. Glaser, C.Y. Ito, A.J. Buckler, J. Pelletier, D.A. Haber, E.A. Rose, A. Kral, H. Yeger, W.H. Lewis, et al. 1990. Isolation and characterization of a zinc finger polypeptide gene at the human chromosome 11 Wilms' tumor locus. Cell. 60:509-520.

7. Lopingco, M.C., and A.S. Perkins. 1996. Molecular analysis of Evi1, a zinc finger oncogene involved in myeloid leukemia. Curr. Top. Microbiol. Immunol. 211:211-222.

8. Dahmane, N., J. Lee, P. Robins, P. Heller, and A. Ruiz i Altaba. 1997. Activation of the transcription factor Gli1 and the Sonic hedgehog signalling pathway in skin tumours. Nature. 389:876-881.

9. Abdollahi, A., D Roberts, A.K Godwin, D.C. Schultz, G Sonoda, and J.R. Testa. 1997. Identification of a zinc-finger gene at 6q25: a chromosomal region implicated in development of many solid tumors. Oncogene. 14:1973-1979.

10. El Rouby, S., and E.W. Newcomb. 1996. Identification of Bcd, a novel proto-oncogene expressed in B-cells. Oncogene. 13:2623-2630.

11. Sauer, F., and H. Jackle. 1991. Concentration-dependent transcriptional activation or repression by Krüppel from a single binding site. Nature. 353:563566 .

12. Sauer, F., J.D. Fondell, Y. Ohkuma, R.G. Roeder, and H. Jackle. 1995. Control of transcription by Krüppel through interactions with TFIIB and TFIIE ß. Nature. 375:162-164.

13. Pengue, G., and L. Lania. 1996. Krüppel-associated box-mediated repression of RNA polymerase II promoters is influenced by the arrangement of basal promoter elements. Proc. Natl. Acad. Sci. USA. 93:1015-1020.

14. Witzgall, R., E. O'Leary, A. Leaf, D. Onaldi, and J.V. Bonventre. 1994. The Krüppel-associated box-A (KRAB-A) domain of zinc finger proteins mediates transcriptional repression. Proc. Natl. Acad. Sci. USA. 91:4514-4518.

15. Margolin, J.F., J.R. Friedman, W.K.-H. Meyer, H. Vissing, H.-J. Thiesen, and F.J. Rauscher III. 1994. Krüppel-associated boxes are potent transcriptional repression domains. Proc. Natl. Acad. Sci. USA. 91:4509-4513.

16. Pengue, G., V. Calabro, P.C. Bartoli, A. Pagliuca, and L. Lania. 1994. Repression of transcriptional activity at a distance by the evolutionarily conserved KRAB domain present in a subfamily of zinc finger proteins. Nucleic Acids Res. 22:2908-2914.

17. Majello, B., P. De Luca, and L. Lania. 1997. Sp3 is a bifunctional transcription regulator with modular independent activation and repression domains. J. Biol. Chem. 272:4021-4026.

18. Klug, A., and J.W. Schwabe. 1995. Protein motifs 5. Zinc fingers. FASEB (Fed. Am. Soc. Exp. Biol.) J. 9:597-604.

19. Bellefroid, E.J., D.A. Poncelet, O.R. Lecocq, and J.A. Martial. 1991. The evolutionarily conserved Krüppel-associated box domain defines a subfamily of eukaryotic multifingered proteins. Proc. Natl. Acad. Sci. USA. 88: 3608-3612.

20. Liu, C., A. Calogero, G. Ragona, E. Adamson, and D. Mercola. 1996. EGR-1, the reluctant suppression factor: EGR-1 is known to function in the regulation of growth, differentiation, and also has significant tumor suppressor activity and a mechanism involving the induction of TGF- $\beta 1$ is postulated to account for this suppressor activity. Crit. Rev. Oncog. 7:101-125.

21. Werner, H., C.T. Roberts, Jr., F.J. Rauscher III, and D. LeRoith. 1996. Regulation of insulin-like growth factor I receptor gene expression by the Wilms' tumor suppressor WT1. J. Mol. Neurosci. 7:111-123.

22. Wang, Z.Y., Q.Q. Qiu, M. Gurrieri, J. Huang, and T.F. Deuel. 1995. WT1, the Wilms' tumor suppressor gene product, represses transcription through an interactive nuclear protein. Oncogene. 10:1243-1247.

23. Gebelein, B., K. Mesa, and R. Urrutia. 1996. A novel profile of expressed sequence tags for zinc finger encoding genes from the poorly differentiated exocrine pancreatic cell line AR4IP. Cancer Lett. 105:225-231.

24. Cook, T., R. Urrutia, and M.A. McNiven. 1994. Identification of dynamin 2, an isoform ubiquitously expressed in rat tissues. Proc. Natl. Acad. Sci. USA. 91:644-648.

25. Cook, T.A., K.J. Mesa, B.A. Gebelein, and R.A. Urrutia. 1996. Upregulation of dynamin II expression during the acquisition of a mature pancreatic acinar cell phenotype. J. Histochem. Cytochem. 44:1373-1378.
26. Santos, E., and A.R. Nebreda. 1989. Structural and functional properties of ras proteins. FASEB (Fed. Am. Soc. Exp. Biol.) J. 3:2151-2163.

27. Blok, L.J., M.E. Grossmann, J.E. Perry, and D.J. Tindall. 1995. Characterization of an early growth response gene, which encodes a zinc finger transcription factor, potentially involved in cell cycle regulation. Mol. Endocrinol. 9:1610-1620.

28. Imoto, M., I. Tachibana, and R. Urrutia. 1998. Identification and functional characterization of a novel human protein highly related to the yeast dynamin-like GTPase VPS1P. J. Cell Sci. 111:1341-1349.

29. Cox, A.D., and C.J. Der. 1994. Biological assays for cellular transformation. Methods Enzymol. 238:277-294.

30. Martin, S.J., C.P.M. Reutelingsperger, A.J. McGahon, J.A. Rader, R.C.A.A. van Schie, D.M. LaFace, and D.R. Green. 1995. Early redistribution of plasma membrane phosphatidylserine is a general feature of apoptosis regardless of the initial stimulus. Inhibition by overexpression of $\mathrm{Bcl}-2$ and $\mathrm{Abl}$. J. Exp. Med. 177:195-200.

31. Schulz, T.C., B. Hopwood, P.D. Rathjen, and J.R.E. Wells. 1995. An unusual arrangement of 13 zinc fingers in the vertebrate gene Z13. Biochem. J. 311:219-224.

32. Glaichenhaus, N., and F. Cuzin. 1987. A role for ID repetitive sequences in growth- and transformation-dependent regulation of gene expression in rat fibroblasts. Cell. 50:1081-1089.

33. Zeleznik-Le, N.J., A.M. Harden, and J.D. Rowley. 1994. 11q23 translocations split the "AT-hook" cruciform DNA-binding region and the transcriptional repression domain from the activation domain of the mixed-lineage leukemia (MLL) gene. Proc. Natl. Acad. Sci. USA. 91:10610-10614.

34. Sadowski, I. 1995. Uses for GAL4 expression in mammalian cells. Genet. Eng. 17:119-148.

35. Bartel, D.P., M. Sheng, L.F. Lau, and M.E. Greenberg. 1989. Growth factors and membrane depolarization activate distinct programs of early response gene expression: dissociation of fos and jun induction. Genes Dev. 3: 304-313.

36. Winkles, J.A. 1998. Serum- and polypeptide growth factor-inducible gene expression in mouse fibroblasts. Prog. Nucleic Acids Res. Mol. Biol. 58:41-78.

37. Bruening, W., P. Moffett, S. Chia, G. Heinrich, and J. Pelletier. 1996. Identification of nuclear localization signals within the zinc fingers of the WT1 tumor suppressor gene product. FEBS Lett. 393:41-47.

38. Xu, N., L. Bradley, I. Ambdukar, and J.S. Gutkind. 1993. A mutant alpha subunit of G12 potentiates the eicosanoid pathway and is highly oncogenic in NIH 3 T3 cells. Proc. Natl. Acad. Sci. USA. 90:6741-6745.

39. Xu, N., T. Voyno-Yasenetskaya, and J.S. Gutkind. 1994. Potent transforming activity of the G13 alpha subunit defines a novel family of oncogenes. Biochem. Biophys. Res. Commun. 201:603-609.

40. Brady, G., R.L. Williams, K. Nordstrom, B. Vennstrom, and N.N. Iscove. 1988. A selectable temperature-sensitive v-src Moloney retrovirus. Oncogene. 3:687-689.

41. Guiochon-Mantel, A., H. Loosfelt, P. Lescop, S. Christin-Maitre, M. Perrot-Applanat, and E. Milgrom. 1992. Mechanisms of nuclear localization of the progesterone receptor. J. Steroid Biochem. Mol. Biol. 41:209-215.

42. Reddy, J.C., and J.D. Licht. 1996. The WT1 Wilms' tumor suppressor gene: how much do we really know? Biochem. Biophys. Acta. 1287:1-28.

43. Friedman, J.R., W.J. Fredericks, D.E. Jensen, D.W. Speicher, X.P. Huang, E.G. Neilson, and F.J. Rauscher III. 1996. KAP-1, a novel corepressor for the highly conserved KRAB repression domain. Genes Dev. 10:2067-2078.

44. Kim, S.S., Y.M. Chen, E. O'Leary, R. Witzgall, M. Vidal, and J.V. Bonventre. 1996. A novel member of the RING finger family, KRIP-1, associates with the KRAB-A transcriptional repressor domain of zinc finger proteins. Proc. Natl. Acad. Sci. USA. 93:15299-15304.

45. Moosmann, P., O. Georgiev, B. Le Douarin, J.P. Bourquin, and W. Schaffner. 1996. Transcriptional repression by RING finger protein TIF1 beta that interacts with the KRAB repressor domain of KOX1. Nucleic Acids Res. 24:4859-4867.

46. Serrano, M., E. Gomez-Lahoz, R.A. DePinho, D. Beach, and D. BarSagi. 1995. Inhibition of ras-induced proliferation and cellular transformation by p16INK4. Science. 267:249-252.

47. Tay, J.S. 1995. Molecular genetics of Wilms' tumor. J. Paediatr. Child Health. 31:379-383.

48. Aubry, M., S. Demczuk, C. Desmaze, M. Aikem, A. Aurias, J.-P. Julien, and G.A. Rouleau. 1993. Isolation of a zinc finger gene consistently deleted in DiGeorge syndrome. Hum. Mol. Genet. 2:1583-1587. 